\title{
Electron density and temperature in the solar corona from multifrequency radio imaging
}

\author{
C. Mercier and G. Chambe \\ LESIA-Observatoire de Paris, CNRS, UPMC, Univ. Paris-Diderot, 92195 Meudon Cedex, France \\ e-mail: claude.mercier@obspm.fr
}

Received 18 December 2014 / Accepted 8 August 2015

\begin{abstract}
Context. The 2D images obtained through rotational aperture synthesis with the Nançay Radioheliograph are suitable for quantitative exploitation. First results are presented.

Aims. We study the variations of the quiet corona in brightness and size during an 8-year period and derive electron density and temperature in the corona.

Methods. Images at 6 frequencies between 150 and $450 \mathrm{MHz}$ for 183 quiet days between 2004 and 2011 were used. Measurements of the brightness temperature $T_{\mathrm{b}}$ beyond the limb allowed coronal density models to be derived in both EW and NS radial directions, with a weak dependence on the electron temperature. The total ranges in the heliocentric distance $r$ are $1.15-1.60 R_{\odot}$ (EW) and 1.0-1.4 $R_{\odot}(\mathrm{NS})$. The agreement between results from different frequencies, in the ranges of $r$ where there is overlapping shows the robustness of the method. The electron temperature, in turn, can be derived from the comparison of the observed mean spectra on the disk with those predicted through transfer calculations from the density models derived from limb observations.

Results. The widths of the brightness profiles that were averaged yearly have minima at cycle minimum (2008-2009). These minima are more pronounced for EW profiles than for NS ones. The derived yearly-averaged density models along equatorial and polar diameters are consistent with isothermal and hydrostatic models. They are characterized by their density value $n_{0}$ extrapolated down to the base of the corona and their scale-height temperature $T_{\mathrm{H}}$. Changes in $n_{0}$ and $T_{\mathrm{H}}$ with solar cycle are given for equatorial and polar regions. The kinetic temperature $T_{\mathrm{e}}$ of electrons in the corona $(\sim 0.62 \mathrm{MK})$ is found to be significantly less than $T_{\mathrm{H}}(\sim 1.5 \mathrm{MK})$. This implies an ion temperature $T_{\mathrm{i}} \sim 2.2 \mathrm{MK}$.

Conclusions. The yearly-averaged variations of these models are less than the dispersion between models derived from other techniques, such as white light and EUV observations, partly because these two techniques are not time-averaged, and they refer to particular days. The radio models are generally less dense, which is compatible with isothermal hydrostatic equilibrium in their range of heliocentric distances, and they show different behaviors with the solar cycle in the equatorial or polar radial directions. The electron kinetic temperature $T_{\mathrm{e}}$ is substantially less than $T_{\mathrm{H}}$.
\end{abstract}

Key words. Sun: radio radiation - Sun: corona

\section{Introduction}

Electron density and temperature are basic variables for describing the solar corona and the physical processes that occur there. In particular, the variations in density with height is a key parameter when modeling the acceleration of the fast solar wind, giving some constraints on mechanical forces and local deposit of energy in the low and medial corona (Lallement et al. 1986; Fludra et al. 1999; Meyer-Vernet 2012).

Before space observations, coronal density measurements relied only on white light observations. Eclipse observations allowed the density to be derived down to low heliocentric distances, e.g. 1.05 times the solar radius $\left(R_{\odot}\right)$ but were restricted to eclipse times. Conversely, ground-based coronographic observations allowed continuous time coverage but were restricted to larger heliocentric distances (typically $>1.2 R_{\odot}$ ). Since the launch of Skylab (May 1973) and, more, recently of SoHO (Dec. 1995), extreme-UV (EUV) and soft X-rays observations were also used to derive coronal densities, with the following limitations: i) they are generally limited to heliocentric distances $<1.2 R_{\odot}$ for the quiet corona near the solar equator, less in coronal holes, and must be completed at greater heliocentric distances with white light observations, now also from space observations; ii) they are restricted to relatively short observational campaigns and have limited statistical significance. Figure 1 gathers the results from various data sets for the heliocentric range $1.0-1.5 R_{\odot}$ and for quiet equatorial and polar regions.

Although not exhaustive, this set of models shows a dispersion of results over about one order of magnitude for both equatorial and polar regions. Moreover, if equatorial densities (Fig. 1 top) show similar decrease with height, this is not the case of polar densities (Fig. 1 bottom), which is possibly because measurements are more difficult since densities are lower. It must also be noted that there are no series of observations done with the same instrument that give the evolution of the coronal density during a large part of a solar cycle. These results show no clear long time-scale trend with the solar cycle phase, for both equatorial and regions. This can be related to their being derived from short observation campaigns and referring to particular (and not mean) states of the corona.

In this paper, we derive electron coronal densities from measurements of the radio brightness beyond the limb in images obtained with the Nançay radioheliograph $(\mathrm{NRH})$ in the range $150-450 \mathrm{MHz}$. As seen later, this allows the coronal 

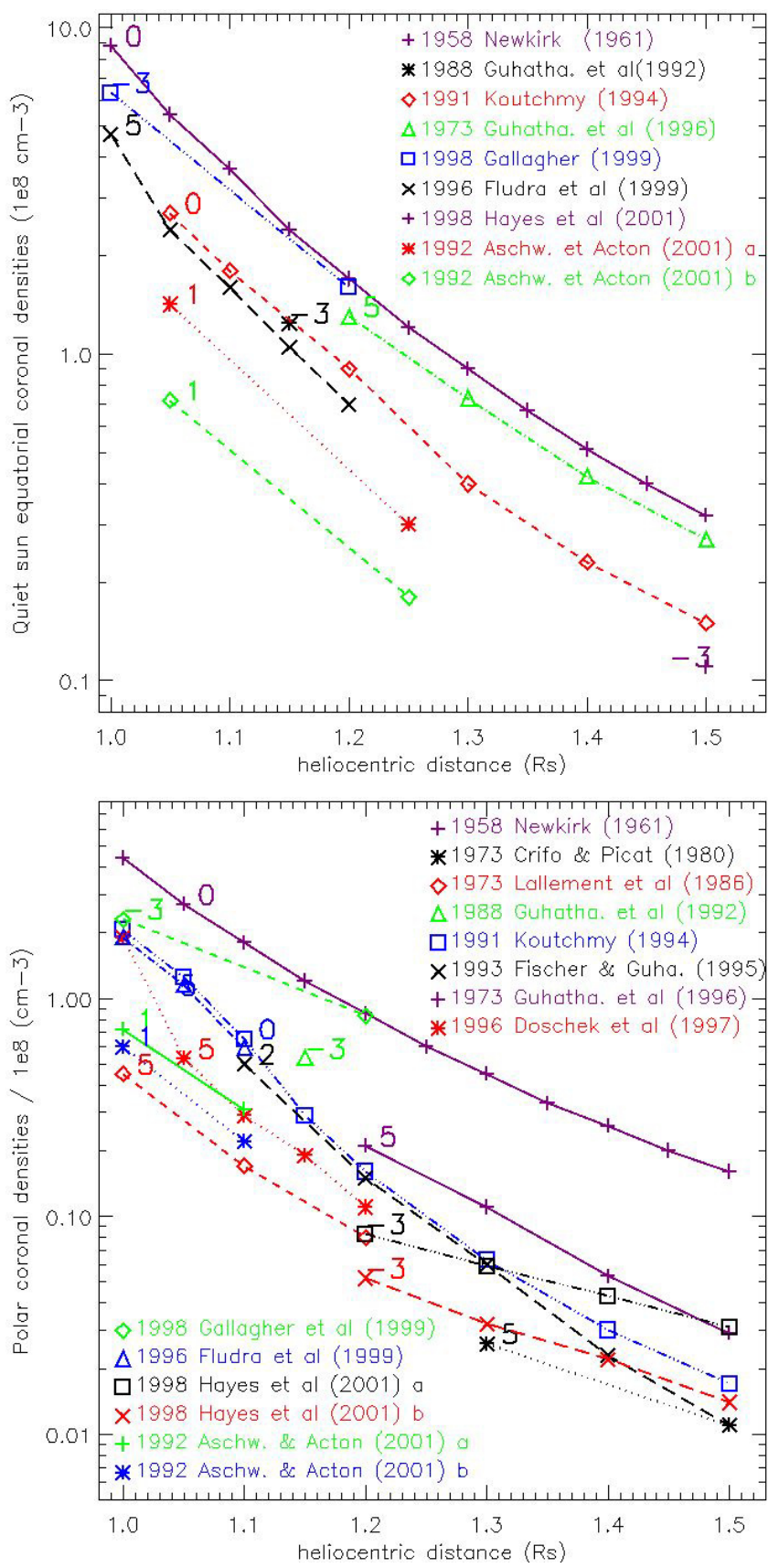

Fig. 1. Density models for quiet corona in equatorial regions (top) and polar regions (bottom). For each set of observations, the time difference relative to the nearest solar maximum is indicated in years, along with the corresponding symbol.

density to be derived at heliocentric distances up to $1.6 R_{\odot}$, which includes both the ranges accessible with EUV observations, which are usually limited to $1.2 R_{\odot}$ and with white-light coronagraphy, usually above $1.3 R_{\odot}$. We give yearly-averaged models of density in equatorial quiet regions and in polar coronal holes for the period 2004-2011, clearly showing an overall evolution during a nearly complete solar cycle. We briefly discuss the significance of the differences with the density models presented in Fig. 1.

\section{Selection of observations and image production}

Reliable brightness measurements beyond the limb require that the images be quantitatively analysed, down to levels that are

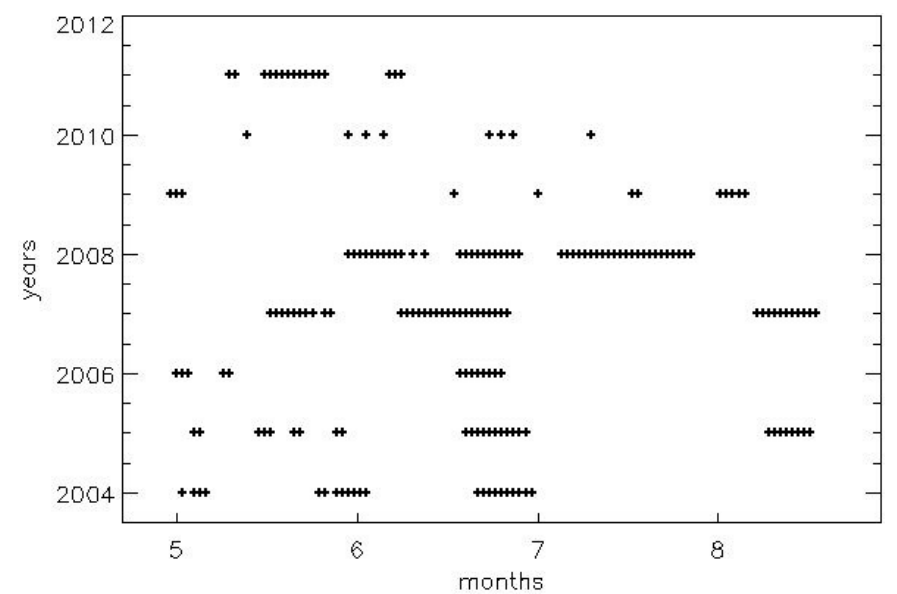

Fig. 2. Selected days. Fractional months of observations are indicated in abscissa.

Table 1. Widths $(\mathrm{EW} \times \mathrm{NS})$ of the NRH field of view for May, 10 (units $R_{\odot}$ ).

\begin{tabular}{lcccc}
\hline \hline Freq $(\mathrm{MHz})$ & 432 & 327 & 236 & 150 \\
\hline Snapshot & $3.0 \times 3.2$ & $4.0 \times 4.2$ & $5.5 \times 5.9$ & $8.6 \times 9.2$ \\
Rot. synth. & $5.0 \times 3.2$ & $6.6 \times 4.2$ & $9.2 \times 5.9$ & $14 \times 9.2$ \\
\hline
\end{tabular}

much lower than those observed on the disk. This requires images with good enough quality and with a field that is much larger than the optical disk. Snapshot NRH images are not suitable for this purpose for several reasons. They suffer from a lack of sensitivity and from interference problems, because of the short integration time. In addition, as explained in Mercier \& Chambe (2009, 2012, hereafter referred to as MC1 and MC2) the instantaneous $u v$-coverage is poor and it limits both the field of view (crucial for for measuring brightness beyond the limb) and the resolution.

For these reasons, we used images obtained with the rotational synthesis technique. These images are better and more reliable at low levels. They have higher resolution and a wider field of view, especially in the EW direction, thanks to the long observation time (up to $7 \mathrm{~h}$ per day). The counterpart is that a low level of non-thermal and variable radio emissions is required, which imply a selection of observations. The images are optimal in summer time, because the elevation of the Sun is too low in winter, so we the used only images for days from May to August. Table 1 displays the EW and NS half-widths of the field of view at four frequencies on May 10 for both rotational synthesis and snapshot images. Values for snapshot images refer to observations at noon, when snapshot images are at best. It will be seen in the following that all these half-widths are larger than the maximum heliocentric distances for which the brightness is measured at the different frequencies, and even much larger at $327 \mathrm{MHz}$ and below. The set of selected data (Fig. 2) is the same as already used by MC2 in their work describing the morphology of the quiet Sun in the range $150-450 \mathrm{MHz}$, but extended to the year 2011. Typical examples of images are displayed in Fig. 3 during the decay, minimum, and growth of solar cycle 23 . Other examples are given by MC1 and MC2. The final data set includes 183 days with none or small non-thermal radio emission during summertime from June 2004 to June 2011. This many days allows to derive yearly-averaged density models. We had images at 150, 164, 236, 327, 410 and $432 \mathrm{MHz}$, 

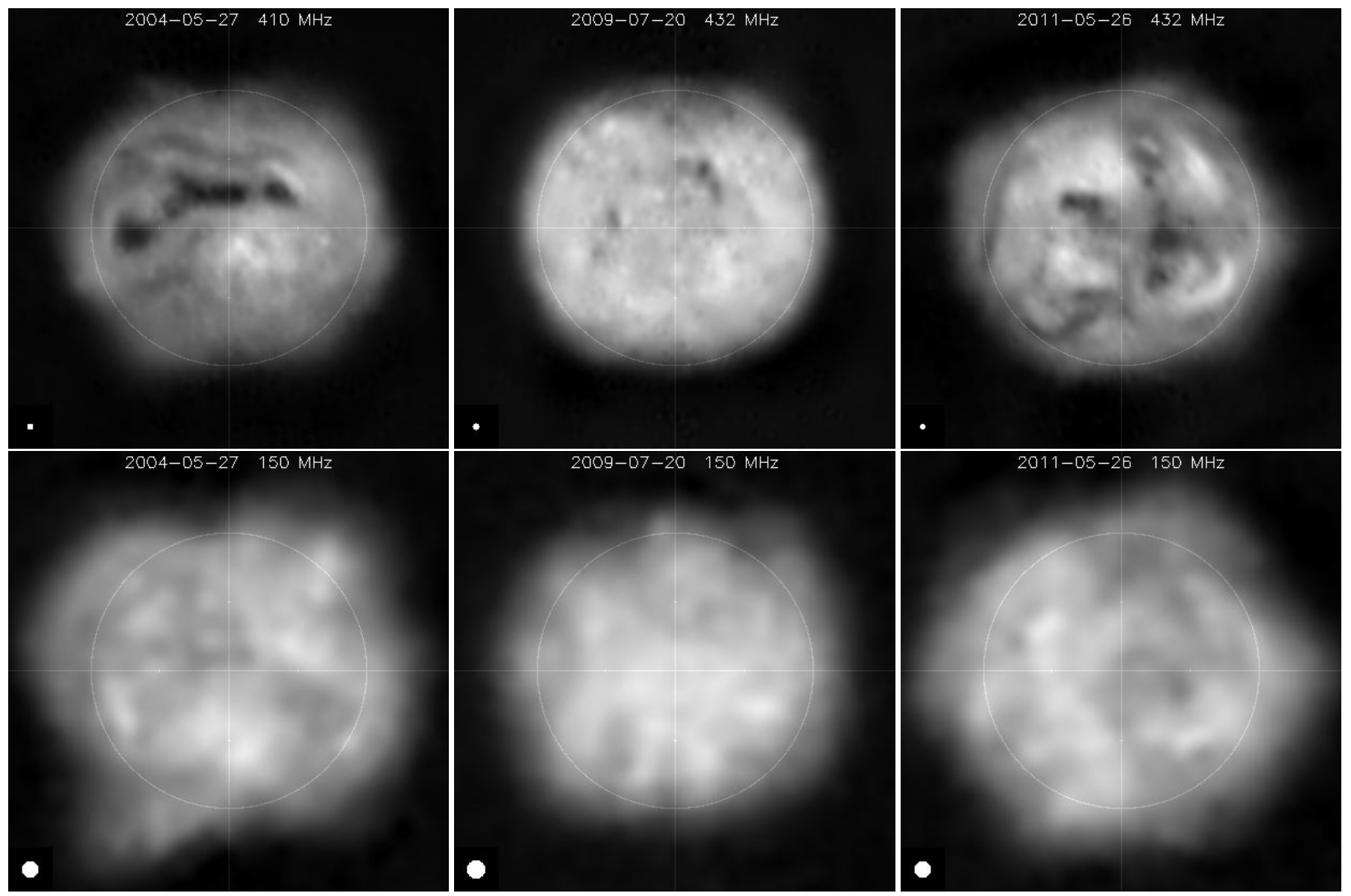

Fig. 3. Examples of images of the Sun. From top to bottom and from left to right: i) during decay of cycle on May 27,2004 , at 410 and $150 \mathrm{MHz}$; ii) during cycle minimum on July 20, 2009 at 432 and $150 \mathrm{MHz}$; and iii) during growth of cycle on May 26, 2011 at 432 and $150 \mathrm{MHz}$. For each day, we note the differences between the aspects of the Sun in high and in low frequencies. The frame widths are $3.2 R_{\odot}$. Resolutions are given at lower left corner. The light circle is the optical limb.

except for $164 \mathrm{MHz}$, which was not used in 2007. The brightness accuracy in images is estimated to $\sim 10-15 \mathrm{kK}$. The steps of the data processing that are specific to the present study are presented after the principle of the method.

\section{Method for deriving coronal densities}

\subsection{Principle of the method}

The method consists in measuring the decrease in the Sun's brightness beyond equatorial and polar limbs. The theory is particularly simple for a density distribution with local spherical symmetry (considering separately equatorial and polar regions), when restricting to brightness values below what is observed on the disk (i.e., for optical depth $\tau$ along the line of sight smaller than unity), and when refraction effects are small. The justification of this last point will be given in Sect. 5.1.

The electron density $n$ has a scale height $H=\frac{k_{\mathrm{B}} T_{\mathrm{H}}}{\bar{m} g} \ll 1 R_{\odot}$, where $k_{\mathrm{B}}$ is the Boltzmann constant, $g$ the local gravity, $\bar{m}=$ $0.6 m_{\mathrm{p}}$ the mean particle mass for helium abundance $0.1, m_{\mathrm{p}}$ is the proton mass, and $T_{\mathrm{H}}$ is a temperature, which may differ from the electron kinetic temperature $T_{\mathrm{e}}$. In a first step, the refraction is not taken into account, and we consider a rectilinear propagation for rays. A simple correction for the (weak) refraction is introduced in a second step.

Neglecting first the refraction, we then consider a straight path with an impact parameter $r_{1}$ in a corona with a spherical electron density distribution. Taking the origin of the coordinate $x$ along this axis at the point of closest approach, the total optical depth along this axis is

$\tau_{1}=\int_{-\infty}^{\infty} \alpha \mathrm{d} x$

where $\alpha=\frac{\xi n^{2}}{\mu f^{2} T_{\mathrm{e}}^{3 / 2}}$ is the absorption coefficient for thermal bremsstrahlung, $\xi=0.23$ (CGS units), $n$ the electron density, $T_{\mathrm{e}}$ the kinetic temperature of electrons, $\mu$ the refractive index, and $f$ the observing frequency. Here and below, the subscript 1 refers to the variable values at $r=r_{1}$,

Since $\alpha$ is proportional to $n^{2}$, the integral in Eq. (1) is essentially restricted to a height range $H_{1} / 2$ above the level $r=r_{1}$. In this narrow layer, we may represent $n$ as $n_{1} \exp -\left(\frac{r-r_{1}}{H_{1}}\right)$ and assume that $T_{\mathrm{e}}=T_{\mathrm{e} 1}$. Writing also $r^{2}=\sqrt{r_{1}^{2}+x^{2}} \simeq r_{1}\left(1+\frac{x^{2}}{2 r_{1}^{2}}\right)$ yields

$\tau_{1}=\frac{\xi}{f^{2} T_{\mathrm{e} 1}^{3 / 2}} n_{1}^{2} \sqrt{\pi H_{1} r_{1}}$

Restricting this to heliocentric distances $r_{1}$ for which $\tau_{1}<1$, we can express the limb brightness temperature $T_{\mathrm{b} 1}=T_{\mathrm{e} 1} \tau_{1}$ as

$T_{\mathrm{b} 1}=\xi \sqrt{\frac{\pi k_{\mathrm{B}}}{\bar{m} g_{1}}} \frac{n_{1}^{2}}{f^{2}} \sqrt{\frac{T_{\mathrm{H} 1}}{T_{\mathrm{e} 1}}}$. 
Measuring the apparent brightness $T_{\mathrm{b} 1}$ then merely yields $n_{1}$ as

$n_{1}=\left(\frac{\bar{m} g_{1}}{\pi k_{\mathrm{B}} r_{1}}\right)^{1 / 4} \frac{1}{\xi^{1 / 2}}\left(\frac{T_{\mathrm{e} 1}}{T_{\mathrm{H} 1}}\right)^{1 / 4} f\left(T_{\mathrm{b} 1}\right)^{1 / 2}$.

In Eq. (4), $T_{\mathrm{e} 1}$ and $T_{\mathrm{H} 1}$ are not known, and they depend on $r_{1}$, but their values are not critical since their ratio appears at quarter power. Thus we assume, in the following, that they take the constant values $T_{\mathrm{e}}$ and $T_{\mathrm{H}}$ over the whole range of the heliocentric distances concerned with our observations. We see below how $T_{\mathrm{e}}$ and $T_{\mathrm{H}}$ can be chosen.

Even if the refractive index $\mu$ is close to unity, the ray paths beyond the limb are not strictly rectilinear. It follows that for actual rays, the distance of closest approach is greater than the impact parameter by an amount that may be not much less than the scale height $H_{1}$. However, as long as the curvature of rays is small (i.e. if their curvature radius $\rho$ is much larger than the heliocentric distance), the integration of $\alpha$ in Eq. (1) along the actual path is close to the integration along a straight line with the same closest approach. In other words, instead of ascribing the density calculated with Eq. (4) to the observed heliocentric distance $r_{1}$, we must ascribe it to the heliocentric distance $r_{\min }$ which corresponds to the closest approach of the ray with the impact parameter $r_{1}$. From Snell's law with spherical symmetry $\mu r \sin (i)=$ cst., where $i$ is the incidence angle on the spherical isodensity layers, it follows that $r_{1} / r_{\min }=\mu\left(r_{\min }\right)$. For weak refraction, $\Delta r$ is $\ll r_{1}$ (but not necessarily $\ll H_{1}$ ) and can be calculated by assuming a density distribution. Using our observations, we can then deduce a new density model. Ideally, this model should coincide with the model used for calculating $\Delta r$. If it is not the case, the procedure should be iterated, taking this new model in order to calculate $\Delta r$. For convenience, we took the density model obtained by neglecting refraction and with $T_{\mathrm{H}}=1.4 \mathrm{MK}$ in the first iteration step. In the subsequent steps, $T_{\mathrm{H}}$ was deduced by fitting the obtained density values to an isothermal and hydrostatic model. The procedure converges in a few steps since the corrections are small.

In summary, Eq. (4) shows that obtaining mean models for the coronal density requires the mean brightness profiles $T_{\mathrm{b}}(r)$ beyond the limb in the EW and NS directions and approximate values of the electron kinetic temperature $T_{\mathrm{e}}$. These variables are obtained as explained in Sects. 3.2 and 3.3.

\subsection{Obtaining mean $1 D E W$ and NS brightness profiles}

Starting with our set of 2D images, the first aim is to produce one-dimensional (1D) profiles along the equatorial and polar axes, averaged over each year of data. The procedure is as follows:

- For each day, equatorial and polar strips are defined with respect to EW and NS directions, as centering on the center of the disk and with half-width $0.3 R_{\odot}$. This width is chosen as a compromise between averaging over several structures and keeping the differences between regions above the equatorial and polar limbs. The $2 \mathrm{D}$ brightness is then averaged over the transverse axes of strips, giving 1D EW and NS profiles for each day.

- Total widths of these daily profiles are measured at 12 brightness levels $T_{\mathrm{b}}$ between 50 and $250 \mathrm{kK}$. The highest value $250 \mathrm{kK}$, is less than the typical value $640 \mathrm{kK}$ chosen below for $T_{\mathrm{b} \text { disk }}$, and this ensures that the condition $\tau_{1}<1$ is fulfilled.
- The yearly-averaged values $\overline{W_{\mathrm{EW}}}\left(T_{\mathrm{b}}\right)$ and $\overline{W_{\mathrm{NS}}}\left(T_{\mathrm{b}}\right)$ of the EW and NS widths for these $T_{\mathrm{b}}$, and the rms dispersions $\sigma_{\mathrm{EW}}$ and $\sigma_{\mathrm{NS}}$ are derived for each frequency.

Because of the width of the EW and NS strips, the derived densities are averages over a solar angle range of about 30 degrees. This must be kept in mind when comparing our results with densities derived from much higher resolution eclipse images that allow, for instance densities to be to derived between polar coronal plumes (e.g., Crifo \& Picat 1980).

\subsection{Obtaining the electron kinetic temperature $T_{\mathrm{e}}$}

The electron kinetic temperature $T_{\mathrm{e}}$ can be tentatively deduced from measurements of the mean brightness on the disk $T_{\mathrm{b} \text { disk }}$ at several frequencies. As seen in Sect. 4.2, $T_{\mathrm{b} \text { disk }}$ increases when the observing frequency $f$ decreases from $445 \mathrm{MHz}$ down to $150 \mathrm{MHz}$ and tends toward a limiting value ranging from 600 to $650 \mathrm{kK}$. The most attractive explanation is that this saturation occurs because $T_{\mathrm{b} \text { disk }} \rightarrow T_{\mathrm{e}}$ when the optical depth $\tau_{\text {cor disk }}$ of the corona on the disk, less than unity at $450 \mathrm{MHz}$, becomes larger than unity at $150 \mathrm{MHz}$ (before it again becomes less than unity at $\sim 60 \mathrm{MHz}$, as can be calculated from standard density models). For each day, $T_{\mathrm{b} \text { disk }}$ was estimated by averaging $T_{\mathrm{b}}$ over a disk of radius $0.9 R_{\odot}$ and by excluding brightest areas around active regions (if any) and dark coronal holes, especially near polar regions. In practice we excluded from regions with brightness temperatures outside the range $250-800 \mathrm{kK}$ and regions at latitudes larger than $60 \mathrm{deg}$. Since the value adopted for $T_{\mathrm{e}}$ in the derivation of the coronal density $n_{\mathrm{e}}$ through Eq. (4) is not critical, we give the results obtained by accepting $T_{\mathrm{e}} \sim 620 \mathrm{kK}$ in Sect. 4.3.

Another hypothesis is that $T_{\mathrm{e}}=T_{\mathrm{H}}$ elsewhere in the corona, meaning that $T_{\mathrm{H}} \sim 1.5 \mathrm{MK}$. Densities derived with this hypothesis are higher merely by a factor $\sim 1.3$, according to Eq. (4). Both possibilities for $T_{\mathrm{e}}$ are discussed in Sect. 5, and it is shown that the hypothesis that $T_{\mathrm{e}}=T_{\mathrm{H}}$ is not tenable.

\section{Results}

\subsection{EW and NS widths of the Sun}

Figure 4 shows the variations in the yearly-averaged apparent EW and NS sizes of the Sun $\overline{W_{\mathrm{EW}}}$ and $\overline{W_{\mathrm{NS}}}$ at several brightness temperature levels and of their rms dispersion for 150, 236, and $432 \mathrm{MHz}$. For clarity, the curves corresponding to high levels $\left(T_{\mathrm{b}}>400 \mathrm{kK}\right)$ or to apparent widths smaller than for the optical disk (not used in this work) are not shown. In spite of the dispersion (indicated by the vertical bars) due to the changes in coronal structures on time scales of some days, the continuous curves clearly show that there is a general trend for both EW and NS widths at all levels toward decreasing in 2004-2005 to a minimum in 2008-2009 and to increasing again in 2010-2011. This time range covers a large portion of solar cycle 23. Similar plots (not shown) for the other NRH frequencies $(164,327$, and $410 \mathrm{MHz}$ ) show the same behavior. This trend is less pronounced for NS profiles at 410 and $432 \mathrm{MHz}$. At these frequencies, the dispersion at low levels is large, which can be partly explained by the limited quality of the images far beyond the limb, mainly because the $u v$-coverage is less dense than at lower frequencies (CM1 and CM2). 
C. Mercier and G. Chambe: Electron density and temperature in the solar corona
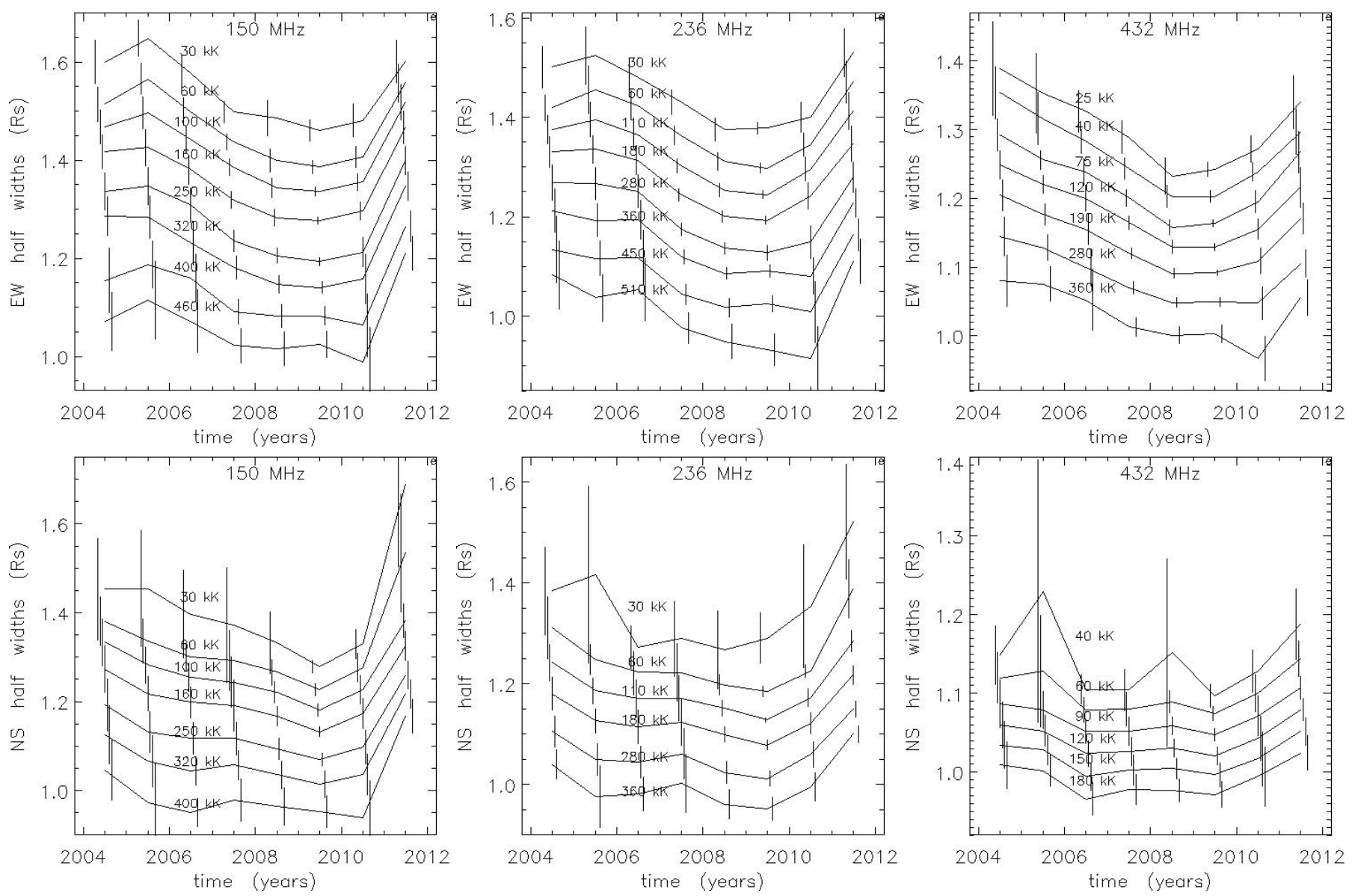

Fig. 4. Apparent widths of the Sun at several levels in EW (top) and NS (bottom) directions for three radio frequencies (150 MHz at left, $236 \mathrm{MHz}$ at middle, $432 \mathrm{MHz}$ at right), between 2004 and 2011. The curves, labeled with the brightness temperature $T_{\mathrm{b}}(\mathrm{kK})$, give yearly-averaged values. The vertical bars give the rms dispersions for each year. They are slightly shifted relative to each other in order not to overlap.
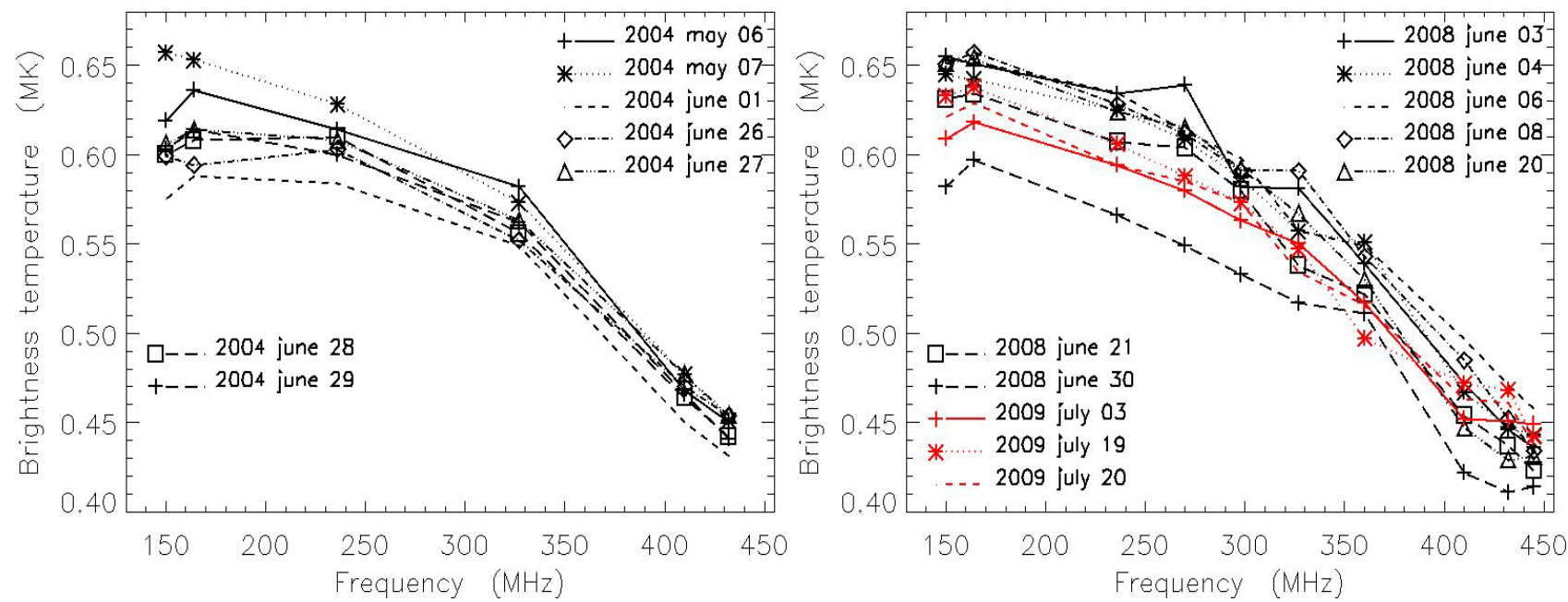

Fig. 5. Examples of variation in the brightness temperature $T_{\mathrm{b} \text { disk }}$ averaged on the disk versus the observing for 7 days in 2004 (just after cycle maximum, left), and for 10 days in 2008-2009 (during cycle minimum, right).

\subsection{Mean brightness on the disk}

Figure 5 displays the variations in $T_{\mathrm{b} \text { disk }}$ versus the observing frequency for several days among the closest ones to the solar maximum (2004 in our data, at left) and during solar minimum (2008-2009, to the right). In all cases, $T_{\mathrm{b} \text { disk }}$ increases with decreasing frequency and tends toward a limiting value in the range $600-650 \mathrm{kK}$. The trend is very similar for both sets, the saturation effect since it is slightly more marked for 2004 than for 2008-2009. The values for $T_{\mathrm{b}}$ are quite similar to those previously derived from radio observations in the same frequency range: Alissandrakis et al. (1985) and Marqué et al. (1999) 
Table 2. Parameters of the equatorial and polar density models derived in Fig. 6.

\begin{tabular}{ccccccc}
\hline \hline Year & $\begin{array}{c}n_{0 \mathrm{EW}} \\
10^{8} \mathrm{~cm}^{-3}\end{array}$ & $\begin{array}{c}\sigma_{n \mathrm{EW}} \\
\%\end{array}$ & $\begin{array}{c}T_{\mathrm{HEW}} \\
\mathrm{kK}\end{array}$ & $\begin{array}{c}n_{0 \mathrm{NS}} \\
10^{8} \mathrm{~cm}^{-3}\end{array}$ & $\begin{array}{c}\sigma_{n \mathrm{NS}} \\
\%\end{array}$ & $\begin{array}{c}T_{\mathrm{HNS}} \\
\mathrm{kK}\end{array}$ \\
\hline 2004 & 3.18 & 7 & 1350 & 0.63 & 10 & 2050 \\
2005 & 1.70 & 10 & 1690 & 0.63 & 9 & 1960 \\
2006 & 1.75 & 10 & 1580 & 0.53 & 9 & 1920 \\
2007 & 1.37 & 8 & 1590 & 0.55 & 9 & 1840 \\
2008 & 1.10 & 7 & 1630 & 0.56 & 7 & 1730 \\
2009 & 1.09 & 8 & 1600 & 0.52 & 8 & 1650 \\
2010 & 1.28 & 8 & 1580 & 0.64 & 8 & 1650 \\
2011 & 1.86 & 12 & 1670 & 0.75 & 12 & 2240 \\
\hline
\end{tabular}

with the NRH, Sheridan \& Mclean (1985) with the Culgoora Radioheliograph. See also the review by Lantos (1998).

Following the discussion in Sect. 3.3, we first adopt $T_{\mathrm{e}} \sim$ $620 \mathrm{kK}$. This value is much lower than the value $T_{\mathrm{H}}$ derived from the scale height with the hypothesis of isothermal and hydrostatic equilibrium $\left(T_{\mathrm{H}} \sim 1.5 \mathrm{MK}\right)$, for both current density models and our models. In Sect. 5, we show that only this low value for $T_{\mathrm{e}}$ can allow predicting spectra on the disk in accordance with those observed (Fig. 5).

\subsection{Derived density models}

Using the mean brightness profiles that correspond to $\overline{W_{\mathrm{EW}}}\left(T_{\mathrm{b}}\right)$ and $\overline{W_{\mathrm{NS}}}\left(T_{\mathrm{b}}\right)$, the coronal densities can be obtained through the procedure described in Sect. 3.1. In doing so, we have taken $r_{1}=\overline{W_{\mathrm{EW}}}\left(T_{\mathrm{b}}\right) / 2$ as the impact parameter for the equatorial case (resp. $r_{1}=\overline{W_{\mathrm{NS}}}\left(T_{\mathrm{b}}\right) / 2$ for the polar case). This results in average models between the models above the $\mathrm{E}$ and $\mathrm{W}$ limbs (resp. $\mathrm{N}$ and $\mathrm{S}$ limbs).

Results for the yearly-averaged coronal densities in equatorial and polar regions over the period 2004-2011 are given in Fig. 6 and summarized in Table 2. Values from the different frequencies are plotted using different symbols and colors. The range in the heliocentric distances $r$ for each frequency is limited: i) downwards by the assumption that $T_{\mathrm{b}}<T_{\mathrm{b} \text { disk }}$, resulting from the assumption $\tau<1$ (in practice $\tau<250 / 620=0.4$ ); and ii) upwards by the lowest level of significant measurement for $T_{\mathrm{b}}$ (in practice $50 \mathrm{kK}$ ). These ranges are shifted towards larger $r$ at decreasing frequencies. They are wide enough to produce partial overlap and then give generally consistent density values. This is particularly true from 2006 to 2009, near the minimum of solar cycle, when the solar brightness distribution was relatively simple. The sets of density values from all frequencies can be fitted with isothermal and hydrostatic models (dotted lines in Fig. 6), characterized by their density $n_{0}$ at the base of the corona and their temperature $T_{\mathrm{H}}$. The rms relative dispersions $\sigma_{n} \mathrm{EW}$ and $\sigma_{n \mathrm{NS}}$ around the fits are also indicated.

There are, however, some cases that show less agreement between frequencies: i) the observed decrease in EW densities derived from 150 and $164 \mathrm{MHz}$ in 2004 is stronger at ii) the rms dispersion around the fits is larger in 2011 (Fig. 6, bottom right). For this last year, two reasons can be given: i) images of the Sun were particularly complex with an increased number of extended structures (due to the growth of solar cycle 24), large brightness contrasts, and different aspects at the various frequencies, making the meaning of averaged brightness profiles more questionable; and ii) instrumental problems, such as imperfect tracking of antennas or interference effects, were more frequent than during the preceding years, reducing the quality of the images. Visual inspection of the images, however, suggests that this effect is less than that the effect of the growth of the solar cycle. It is nevertheless clear that the coronal densities increase significantly between 2010 and 2011 .

It thus can be concluded that using several observing frequencies between 150 and $432 \mathrm{MHz}$ allows coronal densities to be derived over heliocentric distance ranges that are much greater than when using only one observing frequency. Table 2 summarizes the results for the yearly-averaged density models in terms of the corresponding values for $n_{0}$ at the base of the corona and of the scale-height temperatures $T_{\mathrm{HEW}}$ and $T_{\mathrm{HNS}}$ (for equatorial and polar models) derived from fitting the derived densities in Fig. 6 and using a mean electron temperature $T_{\mathrm{e}}=620 \mathrm{kK}$. The rms relative dispersions $\sigma_{n \text { EW }}$ and $\sigma_{n \text { NS }}$ of results around these models are given in percentages. All values for $T_{\mathrm{HEW}}$ and $T_{\mathrm{HNS}}$ are much larger than the value adopted for $T_{\mathrm{e}}$.

\section{Discussion}

\subsection{Curvature of ray paths}

The integration for obtaining the optical thickness along the actual ray path was carried out from the actual minimum approach $r_{\text {min }}$ (not from the apparent one $r_{1}$ ), but neglecting the curvature of the ray. It must now be checked that this assumption is valid. From Snell's law, the local curvature radius $\rho$ of the ray path can be expressed as

$\frac{1}{\rho}=\frac{1}{\mu} \frac{\mathrm{d} \mu}{\mathrm{d} r} \sin (i)$

Setting $n_{f}$ as the electron density corresponding the a plasma frequency equal to the observing frequency $f$, one gets

$\rho=2\left(\frac{n_{f}}{n}-1\right) \frac{H}{\sin (i)}$.

For a given observing frequency $f$, the ratio $n_{f} / n$ increases with heliocentric distance with the same scale height $H$ as the density itself. The ratio $H / \sin (i)$ increases also, although more slowly. Since the layer that essentially contributes to the integration when calculating $\tau$ has a thickness $H / 2$ above the level of closest approach $r_{\min }$, we must evaluate $\rho$ in this layer. Since $H \ll r_{\min }$, we can take $\sin (i) \sim 1$. This leads in fact to a small underestimation of $\rho$ above the level $r=r_{\min }$.

Table 3 displays the results for years 2004 and 2009 at four frequencies. The first row gives the ranges for the heliocentric distance $r$ in the equatorial direction, which is involved in density derivation (units $R_{\odot}$ ). The following rows give the corresponding ranges for the coronal density $n_{\mathrm{e}}$ (units $10^{8} \mathrm{~cm}^{-3}$ ), the refractive index $\mu$, and the radius of curvature $\rho$. The increase in $\rho$ along the ray path in the layer of thickness $H / 2$ can be estimated $\sim 50 \%$ from Eq. (6). Given the values of $\rho$ in Table 3, it follows that the integration of $\alpha$ in Eq. (1) along a straight line leads to overestimating $\tau$ by a few percentage points for the innermost rays (less for higher rays), which leads to underestimating $n_{\mathrm{e}}$ (through Eq. (4)) by the same amount. Furthermore, when fitting the results from several observing frequencies with the same model, this effect is reduced because the ranges of the heliocentric distances overlap.

The situation is quite similar for the derivation of density in polar regions. 
C. Mercier and G. Chambe: Electron density and temperature in the solar corona
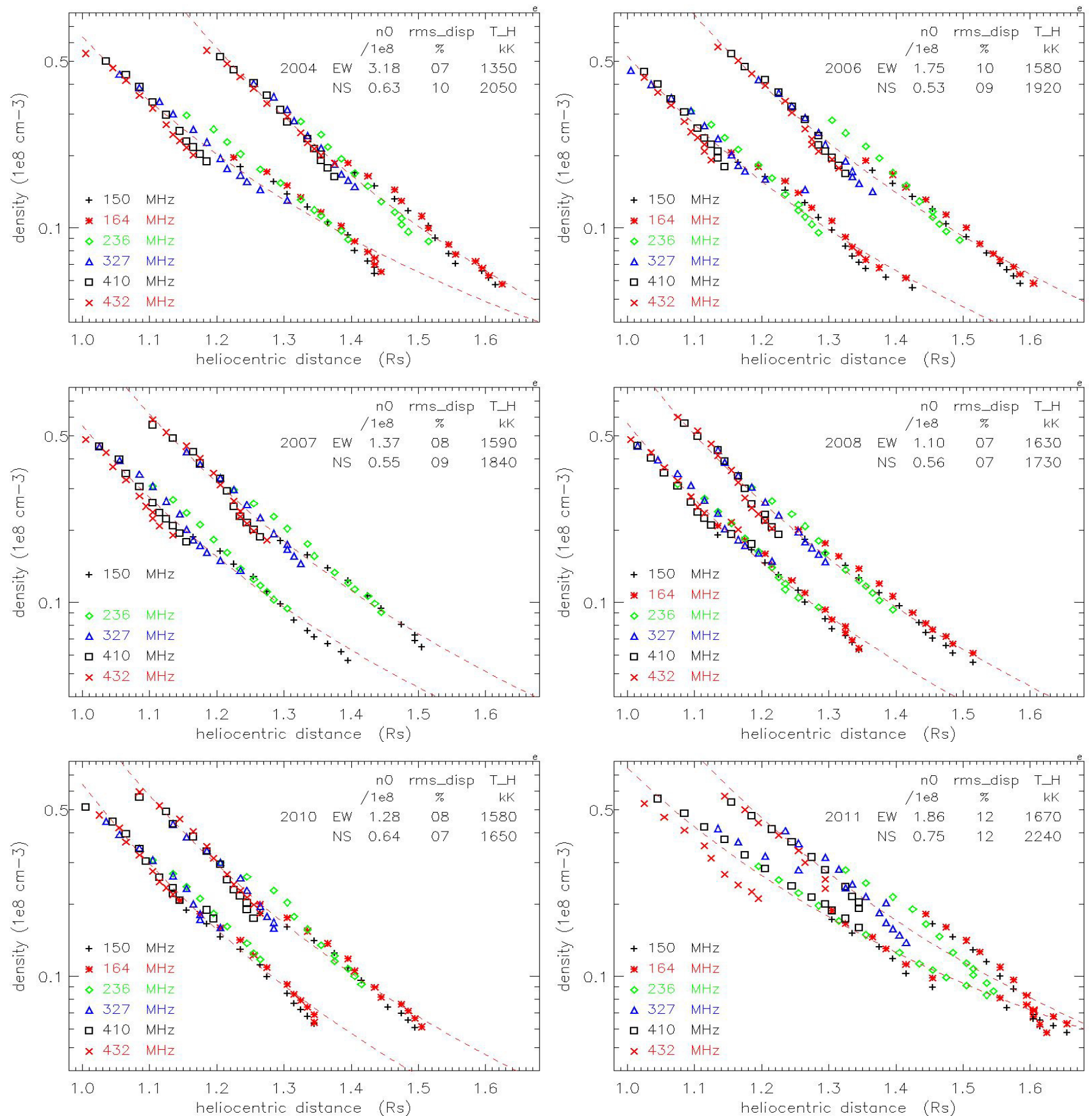

Fig. 6. Densities derived for years 2004-2011 from brightness measurements beyond the EW (top in each frame) and NS (bottom in each frame) limbs. The dashed lines are the best fits for density distributions in isothermal hydrostatic models. The corresponding densities at the base of the corona $n_{0}$, with their relative rms dispersions $\sigma_{n \text { EW }}$ and $\sigma_{n \mathrm{NS}}$, and the hydrostatic temperatures $T_{\mathrm{H}}$ are given for EW and NS density profiles.

\subsection{Mean spectra on the disk and electron kinetic temperature}

In this section we examine the consistency between the value accepted for $T_{\mathrm{e}}(620 \mathrm{kK}$ or $1.5 \mathrm{Mk})$ in deriving density models from brightness measurements beyond the limb, and the mean spectra observed on the disk.

As a preliminary step, we evaluate the optical thickness $\tau_{\mathrm{c}}$ of the corona at the center of the disk: $\tau_{\mathrm{c}}=\int_{r=R_{\odot}}^{\infty} \alpha \mathrm{d} r$, using our equatorial density models and with both hypotheses for $T_{\mathrm{e}}$.
Table 4 displays the results at four frequencies for 2004 (dense corona), 2007, 2008-2009 and 2010 (less dense coronas). The first values refer to $T_{\mathrm{e}}=1550 \mathrm{kK}$. Values in italics between parentheses refer to $T_{\mathrm{e}}=T_{\mathrm{H}}=1550 \mathrm{kK}$. It appears that in both cases, $\tau_{\mathrm{c}}$ at low frequencies largely exceeds unity near the maximum of the cycle (in 2004). If we accept that $T_{\mathrm{e}}=T_{\mathrm{H}}$, it will then be difficult to avoid predicting values for $T_{\mathrm{b}}$ on the order of $1.5 \mathrm{Mk}$. Conversely during the minimum of the cycle (2008-2009), $\tau_{\mathrm{c}} \leq$ unity at low frequencies (and much less at high frequencies) and it will also be difficult to account for the 
Table 3. Ranges for the heliocentric distance $r$ used at four frequencies and the corresponding $n_{\mathrm{e}}, \mu$, and $\rho$ for years 2004 and 2009 (see text).

\begin{tabular}{lcccc}
\hline \hline$f(\mathrm{MHz})$ & 432 & 327 & 236 & 150 \\
\hline 2004 & & & & \\
$r$ & $1.18-1.38$ & $1.25-1.41$ & $1.33-1.52$ & $1.41-1.62$ \\
$n_{\mathrm{e}}$ & $0.67-0.19$ & $0.42-0.17$ & $0.26-0.10$ & $0.17-0.06$ \\
$\mu$ & $0.99-1.00$ & $0.98-0.99$ & $0.98-0.99$ & $0.97-0.99$ \\
$\rho$ & $9-44$ & $9-31$ & $9-31$ & $6-22$ \\
\hline 2009 & & & & \\
$r$ & $1.07-1.24$ & $1.12-1.29$ & $1.20-1.40$ & $1.25-1.48$ \\
$n_{\mathrm{e}}$ & $0.62-0.21$ & $0.43-0.16$ & $0.26-0.09$ & $0.20-0.07$ \\
$\mu$ & $0.99-1.00$ & $0.98-0.99$ & $0.98-0.99$ & $0.96-0.99$ \\
$\rho$ & $10-40$ & $9-32$ & $9-33$ & $5-21$ \\
\hline
\end{tabular}

Table 4. Calculated optical thickness $\tau_{\mathrm{c}}$ of the corona at the center of the disk (see text).

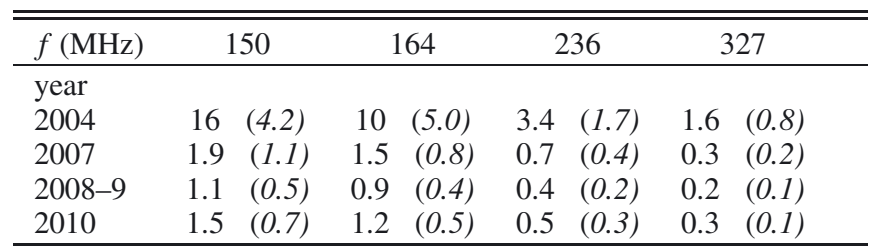

observed saturation of spectra at low frequencies. We examine the situation in more detail in the rest of this section. To this purpose, we now solve the transfer equation using the density models derived from brightness measurements beyond the limb in either case. We recall that these models merely differ by a factor $\sim 1.3$.

In the transfer equation,

$T_{\mathrm{b}}=\int_{\text {ray path }} T_{\mathrm{e}} \mathrm{e}^{-\tau} \mathrm{d} s$

$\tau$ is the current optical thickness and $\mathrm{d} s$ the differential length along the ray path. For the sake of simplicity, we restrict the computation of $T_{\mathrm{b}}$ at the center of the disk, where the ray path is radial.

In contrast to the method presented in Sect. 3.1, where only the corona was concerned, we now need a model for the transition region (TR) since the rays along the line of sight toward the center of the disk can reach the base of the corona, at least at the highest frequencies. Using simple analytical forms (see Appendix), we adopted temperatures profiles such as presented in Fig. 7. These profiles fit with the chromospheric and coronal values at the lower and upper limits of the TR. The thicknesses $h_{1}$ and $h_{2}$ below the inflexion point (Fig. 7) are considered as a free parameters. The density profile $n_{\mathrm{TR}}$ in the TR is then derived from the $T_{\mathrm{H}}$ profile under the assumption of hydrostatic equilibrium:

$\ln \left(\frac{n_{\mathrm{TR}}}{n_{\mathrm{chr}}}\right)=\int_{0}^{z} \frac{1}{T_{\mathrm{H}}}\left(\frac{\bar{m} g}{k_{\mathrm{B}}}+\frac{\mathrm{d} T_{\mathrm{H}}}{\mathrm{d} z}\right) \mathrm{d} z^{\prime}$,

where $n_{\text {chr }}$ is the density at the top of the chromosphere, such as $n_{\mathrm{TR}}$ fits with the density at the base of the corona.

Figures 8 to 12 show the comparison between the observed spectra and the predicted ones. Assuming that $T_{\mathrm{e}}=T_{\mathrm{H}}$ leads to predicted spectra that are quite different from the observed ones (Fig. 8), even for large widths $h_{2}$ of the upper TR, up to $100 \mathrm{Mm}$. In 2004, the spectra saturates at low frequencies since the corona is dense and optically thick, but at high values $\sim 1.5 \mathrm{MK}$. There
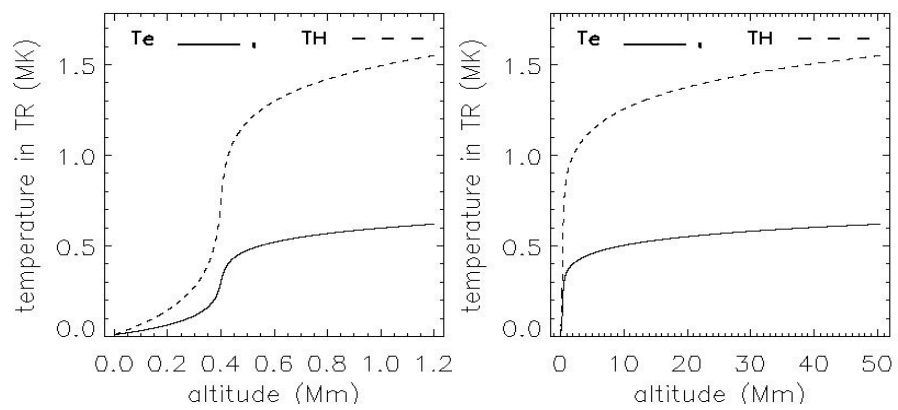

Fig. 7. Examples of the adopted profiles for the electron an the scaleheight temperatures $T_{\mathrm{e}}$ and $T_{\mathrm{H}}$ in the transition region. In the left frame the lower width $h_{1}$ and upper widths $h_{2}$ (resp. below and above the inflexion point of the T profiles) are 0.4 and $0.8 \mathrm{Mm}$. In the right frame, $h_{2}=50 \mathrm{Mm}$.

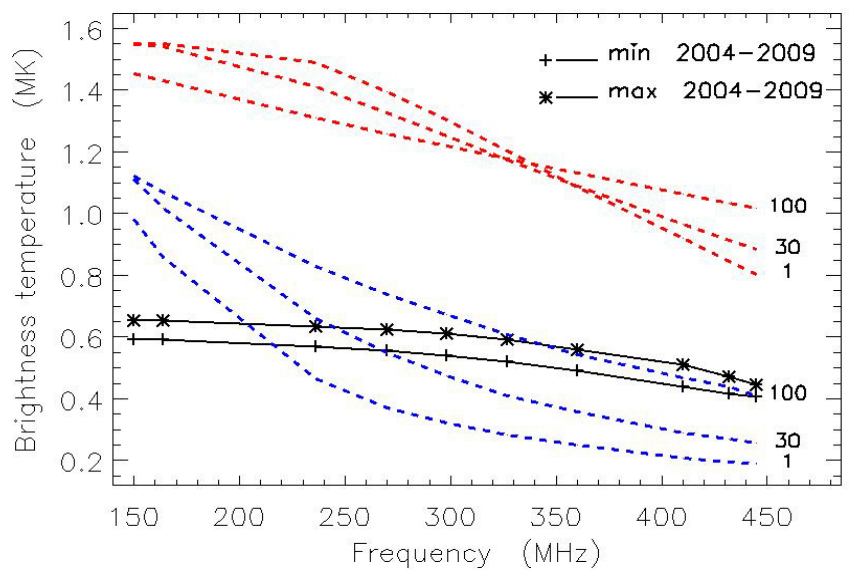

Fig. 8. Comparison between the range of the observed spectra on the disk (between black curves) and the predicted spectra (blue and red curves), assuming that $T_{\mathrm{e}}=T_{\mathrm{H}}=1550 \mathrm{kK}$ elsewhere in the corona. Theoretical curves are labeled with the thickness $h_{2}$ of the upper part of the TR (Mm). Red curves refer to 2004 (near cycle maximum) and blue ones to cycle minimum (2008-2009).

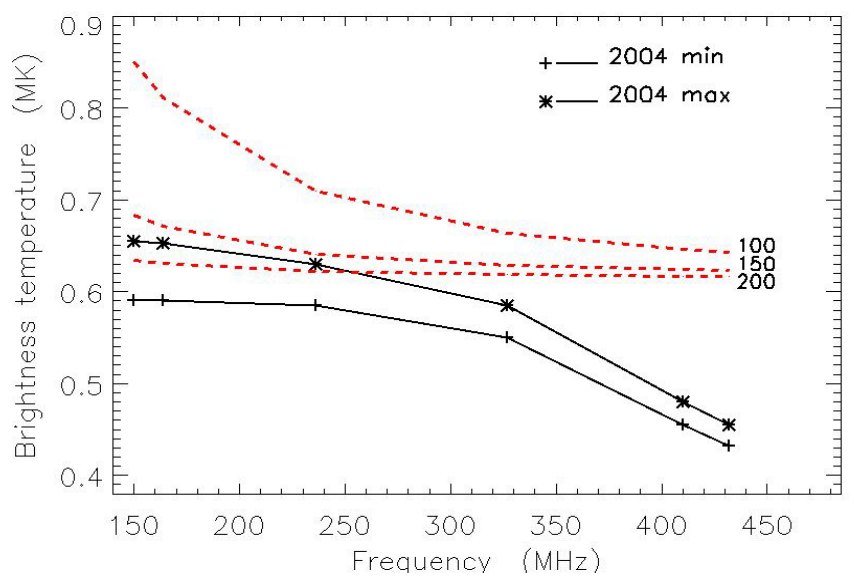

Fig. 9. Same as for Fig. 8 for the predicted spectra in 2004 (dashed curves), assuming that i) the lower part of the corona, with thickness $h_{3}$ is isothermal at $620 \mathrm{kK}$ with thickness $h_{3}$; ii) the temperature in the upper part is $1.5 \mathrm{MK}$; and iii) $T_{\mathrm{e}}=T_{\mathrm{H}}$ elsewhere. Curves are labeled with the thickness $h_{3}(\mathrm{Mm})$.

is no saturation during minimum since the corona is less dense with an optical thickness $\tau_{\mathrm{c}}<1$ at the center of the disk, but the predicted values for $T_{\mathrm{b}}$ are also much more than observed. The reason is that most of the radiation originates in the corona 
C. Mercier and G. Chambe: Electron density and temperature in the solar corona

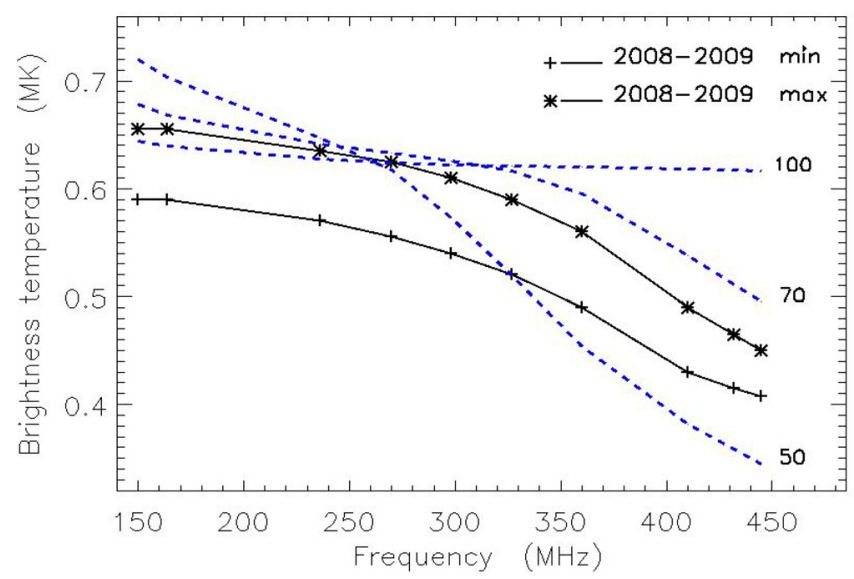

Fig. 10. Same as for Fig. 9 for 2008-2009.

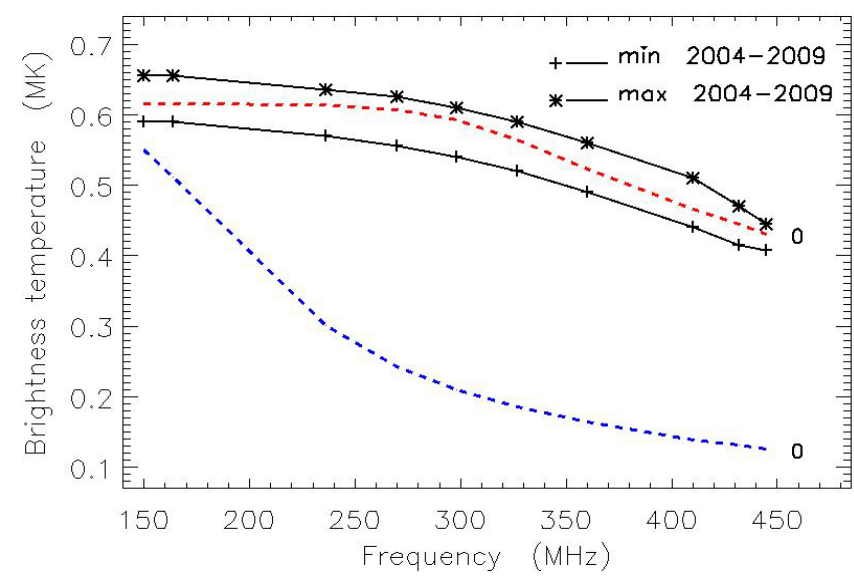

Fig. 11. Comparison between the range observed spectra on the disk in 2004-2009 (indicated by the black curves) and the predicted spectra for 2004 (red curve) and 2008-2009 (blue curve), assuming $T_{\mathrm{e}}=$ $615 \mathrm{kK}$ and $T_{\mathrm{H}}=1.5 \mathrm{MK}$.

itself or from the upper part of the transition region, where the temperature is high.

It follows that the only way to keep the hypothesis $T_{\mathrm{e}}=T_{\mathrm{H}}$ is to assume that the lower part of the corona is quasi-isothermal at a lower temperature, approximately equal to the observed saturation value $620 \mathrm{kK}$ of spectra in Fig. 5. The coronal density at high altitudes is constrained by our observations, and the density at lower levels can be deduced by assuming continuity of density or pressure at the base of the hot upper corona. Assuming again that $T_{\mathrm{e}}=T_{\mathrm{H}}$ elsewhere in the corona and taking the thickness $h_{3}$ of lower layer as a free parameter, we produced the predicted spectra for 2004 and 2008-9. Results are shown in Figs. 9 and 10. The values for $h_{3}$ in Figs. 9 and 10 were selected in such a way that the predicted $T_{\mathrm{b}}$ at $150 \mathrm{MHz}$ are comparable to the observed values. They are large in order to make the upper hot corona optically thin and to reduce its contribution. The fair disagreement between the observed and predicted spectra (even for high values of $h_{3}$, so inconsistent with our limb observations) leads us to also rule out the hypothesis $T_{\mathrm{e}}=T_{\mathrm{H}}$ in the case of a corona with a lower layer at lower temperature.

We now investigate the case $T_{\mathrm{e}} \neq T_{\mathrm{H}}$, with $T_{\mathrm{e}}=620 \mathrm{kK}$ (suggested by the observed spectra on the disk) and $T_{\mathrm{H}}=$ 1.5 MK (from the limb observations). The results for 2004 and 2008-2009 are displayed in Fig. 11. The agreement is satisfactory for 2004, but it is clear that in 2008-2009 a contribution

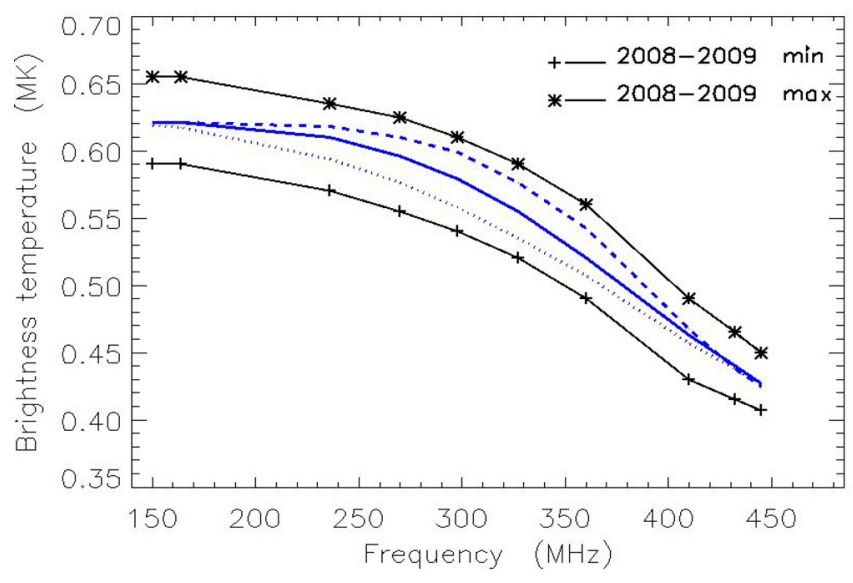

Fig. 12. Comparison between the range of the observed spectra on the disk for 2008-2009 (between black curves) and the predicted spectra, assuming two coronal layers with $T_{\mathrm{H}}=615 \mathrm{kK}$ and $1.5 \mathrm{MK}$, and with $T_{\mathrm{e}}=615 \mathrm{kK}$ elsewhere in the corona (blue curves). The continuous line corresponds to $h_{3}=20 \mathrm{Mm}$ and $h_{2}=10 \mathrm{Mm}$, the dotted line to $h_{3}=10 \mathrm{Mm}$ and $h_{2}=28 \mathrm{Mm}$ and $\mathrm{Mm}$, and dashed line to $h_{3}=28 \mathrm{Mm}$ and $h_{2}=1.7 \mathrm{Mm}$.

of denser layer is lacking for obtaining a saturation of the predicted spectrum at low frequencies. We thus assumed, as above, a low layer in the corona with $T_{\mathrm{e}}=T_{\mathrm{H}}=620 \mathrm{kK}$ and an upper layer with the same $T_{\mathrm{e}}$ but with $T_{\mathrm{H}}=1.5 \mathrm{MK}$, as indicated by the observations. The smaller scale height in the low coronal layer results in higher densities at low levels because of the continuity of density or pressure at the base of the upper corona.

Assuming first the continuity of pressure, the resulting predicted spectra for 2008-2009 are displayed in Fig. 12 for three combinations of the height $h_{3}$ of the cold corona and of the height $h_{2}$ of the upper TR, chosen in such a way that the predicted $T_{\mathrm{b}}$ at $445 \mathrm{MHz}$ are in the middle of the observed range. The agreement with the behavior of the observed spectra is now satisfactory for a relatively narrow range of $h_{3}$ (between 20 and $30 \mathrm{Mm}$ ) and is better for $h_{3}=20 \mathrm{Mm}$ and $h_{2}=10 \mathrm{Mm}$. Assuming the continuity of density leads to quite similar spectra, but with somewhat higher values for $h_{3}$ between 55 and $70 \mathrm{Mm}$ (better fit for $65 \mathrm{Mm}$ ) since the lower corona is less dense at its top. These values for $h_{3}$ are consistent with our observations (down to heights $\sim 90 \mathrm{Mm}$ above the equatorial limb) since a higher value should have been revealed by our observations above the EW limb. Given the limited thickness of this cooler and denser coronal layer, the densities therein are comparable to those in the maximum corona at the same altitudes. We are thus led to the picture of the corona where the density is reduced during cycle minimum, but relatively less in a very thin low layer.

It is of interest to compare these results with the BaumbachAllen density model (Allen 1947):

$n_{\mathrm{e}}=10^{-8} \times\left(2.99 r^{-16}+1.55 r^{-6}\right)$

where $r$ is in $R_{\odot}$ units. The ratio of the scale heights corresponding to the two terms (or equivalently to their associated scaleheight temperatures) is merely the ratio of the exponents $16 / 6$, which is very close to the ratio $1550 / 620$ we adopted for $T_{\mathrm{H}} / T_{\mathrm{e}}$. In addition the altitude above which the second term exceeds the first one is $47 \mathrm{Mm}$, which is intermediate between the thicknesses $h_{3}=20$ or $65 \mathrm{Mm}$ of the lower corona we need to reproduce the spectrum on the disk in 2008-2009.

We conclude that the coronal densities derived from brightness observations beyond the limb, which are independent of the 
structure of the very low corona, and the mean spectra measured on the disk, which involve this very low corona, can be reconciled only if $T_{\mathrm{e}}$ and $T_{\mathrm{H}}$ are different, except at low altitude $(\sim 50 \mathrm{Mm})$ during minimum, where denser regions are needed to produce a saturation of spectra at low frequencies, because the density at higher levels is too low to make the optical thickness $\tau$ of the corona large enough to produce a saturation effect. Such a denser coronal layer, which is limited to low levels, could result frmm magnetic confining in low-altitude closed loops.

That $T_{\mathrm{e}}<T_{\mathrm{H}}$ affects the ion temperature $T_{\mathrm{i}}$, since the observed scale height of the density must be explained. Wave pressure due to dissipation of Alfven waves has been considered in addition to kinetic pressure forces for calculating the coronal density profiles (Zhao 1981), but only beyond $2 R_{\odot}$. At lower altitudes, $T_{\mathrm{HEW}}$ and $T_{\mathrm{HNS}}$ cannot be higher than the mean plasma temperature. The only possible exception is the special case of concentric closed magnetic loops in which the density at the base increases with the size of the loops, resulting in localized coronal cavities. However, this possibility cannot be called upon for the whole quiet Sun and even in coronal holes where the field is radial. It follows that the relatively low value of $T_{\mathrm{e}}$ must be compensated for by a higher temperature $T_{\mathrm{i}}$ for protons and ions $\mathrm{He}^{2+}$. To obtain a scale-height temperature $T_{\mathrm{H}}=1.5 \mathrm{MK}$ and an helium abundance $a=0.1$, a ion temperature $T_{\mathrm{i}}=2.2 \mathrm{MK}$ is needed.

\subsection{Coronal densities and scale-height temperature}

The sets of density values derived for the different frequencies fit together reasonably well (Fig. 6), which gives confidence in the results. Given the limited quality of results, the overall variations in the coronal density with the heliocentric distance $r$ is consistent with an isothermal and hydrostatic model, so there is no need to consider a more sophisticated one.

Equatorial coronal densities are derived in the range $1.2<$ $r<1.6 R_{\odot}$ (down to $1.15 R_{\odot}$ in 2009 , at cycle minimum) and the polar densities in the range $1.0<r<1.4 R_{\odot}$. The equatorial densities are not biased by the presence of dense structures related to active regions and usually restricted to low altitudes. These densities are thus representative of the mean quiet Sun and can include the contribution of streamers or pseudo-streamers at low latitudes, which are in fact parts of the quiet Sun. There are no such contributions for the polar densities.

The polar densities are lower than the EW ones by a factor $\sim 2$, and their scale heights, hence their $T_{\mathrm{H}}$, are slightly larger (see Fig. 6 and Table 1). This can be partly explained by the fact that magnetic fields are totally open in polar regions, whereas large-scale closed field lines can confine the hot plasma in equatorial regions.

\subsection{Changes during solar cycle}

The changes in the yearly-averaged densities show overall trends with the phase of solar cycle. In equatorial regions, the main changes concern the density $n_{0}$ at the base of the corona, with a decrease by a factor of nearly 3 between 2004 and the marked minimum in 2008-2009, followed by a rapid increase in 2010 and particularly in 2011. In contrast, the scale height does not show significant variations.

In polar regions, the behavior is the opposite: the density $n_{0}$ at the base of the corona (directly measured since the corona remains optically thin just above the limb) changes by less than $20 \%$, whereas the scale-height temperature $T_{\mathrm{H}}$ decreases by $\sim 350 \mathrm{kK}$ between 2004 and the cycle minimum, then sharply increases by $\sim 400 \mathrm{kK}$ between 2009 and 2011. As a consequence, the density in polar regions begins to resemble the equatorial ones in 2011 at heliocentric distances $\sim 1.5 R_{\odot}$ (Fig. 6, bottom right).

\subsection{Comparison with previously published results}

\subsubsection{Coronal temperatures}

It is already known that several temperatures can be defined in the corona, depending on the used diagnostics. In addition to the temperature formally deduced from the observed scale height, kinetic temperatures $T_{\mathrm{e}}$ and $T_{\mathrm{i}}$ can be defined for electrons and ions (reviewed by Noci 2003). The degree of agreement (or disagreement) between the different values of $T_{\mathrm{e}}$ and $T_{\mathrm{i}}$ derived from different diagnostics has evolved during recent decades (Noci 2003).

The values for $T_{\mathrm{e}}$ derived from radio observations ( $\left.\sim 0.7 \mathrm{MK}\right)$ are still somewhat lower than those derived from EUV observations, which range from 1.0 to $1.35 \mathrm{MK}$ and may depend on the nature of the lines used (Noci 2003). Thus the agreement between radio and EUV values for $T_{\mathrm{e}}$ is still an open question.

The EUV diagnostics indicate that $T_{\mathrm{i}}>T_{\mathrm{e}}$. It is now accepted that the difference is real and is possibly due to ion heating through cyclotron resonance with transverse waves propagating along the magnetic field. From SUMER off-disk observations, Landi (2007) and Landi \& Cranmer (2009) found that $T_{\mathrm{i}}$ exceeds $T_{\mathrm{e}}$ by factors up to 2.5 , particularly near cycle maximum and with no dependence on the heliocentric distance (within the field of view of SUMER, limited to $\sim 1.3 R_{\odot}$ ), confirming earlier results (references in Landi 2007).

We note that if the value we derive for $T_{\mathrm{e}}$ from radio observations is lower than those derived from the EUVs, there is better agreement between our $T_{\mathrm{i}}$ value of $2.2 \mathrm{MK}$ derived by assuming hydrostatic equilibrium and the $T_{\mathrm{i}}$ values from EUV observations.

\subsubsection{Coronal densities}

The density models derived from EUV are usually restricted to less than $1.2 R_{\odot}$ (especially in polar holes), and those from coronographic observations are usually restricted above $2.0 R_{\odot}$. Our results concern an intermediate height range, which can thus be covered by using the same radio technique. Furthermore, EUV diagnostics involve complicated atomic physics and are sensitive to departure from thermodynamic equilibrium, whereas the radio diagnostic involves only free-free absorption/emission in the coronal plasma, with a simple transfer as long as the transition region is not concerned, which is the case for observations beyond the limb or on the disk at frequencies below $200 \mathrm{MHz}$, for which the corona is optically thick.

For equatorial densities, our models are among the less dense ones displayed in Fig. 1 (top), and they have similar scale heights. For polar densities, the situation is more complex. The models displayed in Fig. 1 (bottom) show more scattered scale heights, which are generally larger above $1.2 R_{\odot}$. Our models have larger and more regularly varying scale heights. They are consistent with isothermal hydrostatic equilibria in the whole range $0<r<1.4 R_{\odot}$ with relatively high values of $T_{\mathrm{H}}$, which regularly change over the period 2004-2011. They are thus among the less dense models below $1.2 R_{\odot}$, and practically the densest ones above $1.2 R_{\odot}$ if we except the Newkirk's model, which is even denser by a factor $\sim 4$. The observed long-timescale behavior may be related to the way we proceeded: i) the 
same technique with the same instrument was used for eight years and ii) yearly-averaged density values were obtained, in contrast to results with various instruments and from eclipses or short campaigns of EUV observations.

\section{Summary and conclusion}

The 2D images of the quiet Sun obtained through rotational synthesis with the NRH are now suitable for quantitative measurements. We showed that off-limb brightness measurements can easily provide coronal density values if we restrict them to brightness levels below those on the disk. Within this limit, the refraction is weak, and taking it into account only results in coronal densities higher by $\sim 30 \%$. The densities derived from the different NRH frequencies between 150 and $450 \mathrm{MHz}$ are obtained in altitude ranges that partially overlap and coincide reasonably well in the common altitude ranges. This allows densities to be obtained in the total ranges $1.15<r<1.6 R_{\odot}$ and $1.0<r<1.4 R_{\odot}$ in equatorial and polar regions, respectively. These ranges overlap with those corresponding to densities derived from EUV observations (mainly below $1.20 R_{\odot}$ ) and those from white light observations (mainly above $1.20 R_{\odot}$ ). Comparing the mean spectra observed on the disk and those derived from our models, we were led to reject the hypothesis that $T_{\mathrm{e}}=T_{\mathrm{H}}$ in the corona, which would involve unrealistically high brightness temperatures $T_{\mathrm{b}}$ on the disk, with non-observed variations with frequency. We found that our models and the value $T_{\mathrm{e}} \sim 620 \mathrm{kK}$ can satisfactorily account for the mean spectra on the disk near cycle maximum, while a relatively thin $(\sim 30 \mathrm{Mm})$ coronal layer with a lower scale height temperature (hence with a higher density) is needed to properly account for the spectra during cycle minimum. Such a cooler layer is consistent with the Baumbach-Allen model (Allen 1947) at low altitudes.

Using a large data base from 2004 to 2011, we gave yearlyaveraged density models for equatorial and polar regions, averaged over latitude ranges that are $\sim 30$ degrees wide. These models are consistent with isothermal hydrostatic equilibrium and can be described by their density $n_{0}$ at the base of the corona and their scale-height temperature $T_{\mathrm{H}}$.

For equatorial regions, $n_{0}$ decreases ftom $2.8 \times 10^{8} \mathrm{~cm}^{-3}$ in 2004 down to $1.1 \times 10^{8} \mathrm{~cm}^{-3}$ at cycle minimum in 2009 , then increases to $1.9 \times 10^{8} \mathrm{~cm}^{-3}$ in 2011 , with $T_{\mathrm{H}}$ remaining between 1.4 and 1.6 MK. For polar models $n_{0}$ shows smaller relative variations (between 0.6 and $0.7 \times 10^{8} \mathrm{~cm}^{-3}$, while $T_{\mathrm{H}}$ decreases from 1.9 MK down to $1.65 \mathrm{MK}$, and then increases to $2.2 \mathrm{MK}$.

When compared to previous models i) our equatorial models are among the least dense ones, with a similar scale height; and ii) our polar models have a larger scaleheight. It follows that they are among the less dense ones below $1.2 R_{\odot}$, and are relatively denser above $1.2 R_{\odot}$. iii) Both equatorial and polar models show simple variations during the solar cycle.

Finally, the relatively wide heliocentric distance range (1.0-1.4 $R_{\odot}$ ) over which our polar models are defined could make them useful for constraining acceleration models of fast solar winds acceleration models including wave pressure and/or non-thermal energy deposit in the low and medium corona.

Acknowledgements. We acknowledge the referee for constructive suggestions.

\section{Appendix}

We seek to find a simple analytical expression for describing the sigmoid-like temperature profiles in the transition region (TR). We first choose the temperature $T_{\mathrm{i}}$ corresponding to the inflection in the curve $T(z)$ and the thicknesses $h_{1}$ and $h_{2}$ of the TR below this inflection (taken here for convenience as the origin of the altitude $z$ ). Let $f_{2}(z)=\left(z / h_{2}+\epsilon\right)^{1 / m}$, where $\epsilon \ll 1$ and $m \gg 1$. When $z$ increases from 0 to $h_{2}, f_{2}(z)$ increases first sharply (but with a finite slope) from $\epsilon^{1 / m}$, then slower and slower toward $\sim 1$. It can be normalized to define temperature profiles between $T_{\mathrm{i}}$ and the coronal temperatures $T_{\mathrm{e}}$ and $T_{\mathrm{H}}$.

In the same way, $f_{1}(z)=\left(-z / h_{1}+\epsilon\right)^{1 / m}$ can be used to define temperature profiles between $T_{\mathrm{i}}$ and the chromospheric temperature $T_{\text {chr }}\left(T_{\text {chr }} \ll T_{\mathrm{i}}\right)$. Smoothing the total resulting profile between $-h_{1}$ and $h_{2}$ in order to get a regular connection at $z=0$, produces curves such as shown in Fig. 7. We used typical values $T_{\mathrm{i}} \sim 0.3 \mathrm{MK}, \epsilon=5 \times 10^{-3}$, and $m=10$. The choices for $T_{\mathrm{chr}}$ and $T_{\mathrm{i}}$ are not critical.

\section{References}

Alissandrakis, C. E., Lantos, P., \& Nicolaidis, E. 1985, Sol. Phys., 97, 267 Aschwanden, M. J., \& Acton, L. W. 2001, ApJ, 550, 475

Allen, C. W. 1947, MNRAS, 107, 426

Crifo F., \& Picat J.-P. 1980, A\&A, 1, 239

Doschek, G. A., Warren, H. P., Laming, J. M., et al. 1997, ApJ, 482, L109

Fischer, R., \& Guhatakurta, M. 1995, ApJ, 447, L139

Fludra, A., del Zanna, G., Alexander, D., \& Bromage, B. J. I. 1999, J. Geophys. Res., 104, 9709

Gallagher, P. T., Mathioukadis, M., Keenan, F. P., Philips, K. J. H., \& Tsinganos, K. 1999, ApJ, 524, L133

Guhathakurta, M., Rottman, G. J., Fischer, R. R., Orall, F. Q., \& Altrock, R. C. 1992, ApJ, 388, 633

Guhathakurta, M., Holzer, T. E., \& MacQueen, R. M. 1996, ApJS, 106, 143

Hayes, A. P., Vourlidas, A., \& Howard, R. A. 2001, ApJ, 548, 1081

Koutchmy, S. 1994, Adv. Space Res., 4, 29

Lallement, R., Holzer, T. E., \& Munro, R. H. 1986, J. Geophys. Res., 91, 6751

Landi, E. 2007, ApJ, 663, 1363

Landi, E., \& Cranmer, S. R. 2009, ApJ, 691, 794

Lantos, P. 1998, Proc. Nobeyama Symp., NRO Report 479

Marqué, C., Lantos, P., Delouis, J.-M., \& Alissandrakis, C. E. 1999, 8th SOHO Workshop: Plasma Dynamics and Diagnostics in the Solar Transition Region and Corona. Proceedings of the Conference held 22-25 June in CAP 15, 1-13 quai de Grenelle, 75015 Paris, France

Mercier, C., \& Chambe, G. 2009, ApJ, 700, L137

Mercier, C., \& Chambe, G. 2012, A\&A, 540, A18

Meyer-Vernet, V. 2012, Basics of the Solar Wind (Cambridge University Press) Newkirk, G. 1961, ApJ, 133, 983

Noci, G. 2003, Mem. Soc. Astron. It., 74, 704

Sheridan, K. V., \& McLean, D. J. 1985, in Solar Radiophysics (Cambridge University Press), 455

Zhao, X.-P. 1981, J. Geophys. Res., 86, 7480 